\title{
Evaluación de experiencias formativas en enfermeria para el cuidado de personas mayores
}

\section{Evaluation of Nursing training experiences for the care of the elderly}

\author{
Carlos Alberto Fernández-Silva ${ }^{1}$ iD; María Paz Sánchez-Sepúlveda ${ }^{2}$ (D)
}

Forma de citar: Fernández-Silva CA, Sánchez Sepulveda MP. Evaluación de experiencias formativas en enfermería para el cuidado de personas mayores. Salud UIS. 53: e21015. doi: https://doi.org/10.18273/saluduis.53.e:21015 @) (1)

\section{Resumen}

Introducción: la formación de pregrado en enfermería requiere del desarrollo de prácticas pedagógicas en las que se incorporen aspectos que permitan fomentar sus características como profesión, disciplina y arte. Objetivo: analizar los resultados de la evaluación realizada a un grupo de estudiantes de enfermería respecto a experiencias formativas en el contexto de una asignatura donde se aborda el cuidado de personas mayores en una universidad pública en la Región de Los Lagos en Chile. Metodología: se implementó una pauta de valoración clínica con el uso de lenguaje estandarizado y el desarrollo de un trabajo artístico; al finalizar la asignatura se desarrolló la evaluación cuanti-cualitativa de dicha experiencia, a través de un cuestionario y la realización de grupos focales y entrevistas individuales. De un curso de 22 estudiantes, 18 respondieron el cuestionario y 8 participaron en grupos focales y entrevistas individuales. Resultados: la experiencia en general fue valorada positivamente por los estudiantes, se evidenció que el $80 \%$ refirió que la pauta de valoración empleada facilitó la aplicación del proceso de enfermería. Respecto del trabajo artístico se apreciaron las estrategias metodológicas que permitieron el desarrollo de este y el aporte que el trabajo representó para la gestión del cuidado, la asignatura y los ámbitos personales. Discusión: se evidencia los aportes que podría tener la metodología empleada en aspectos como la resolución de problemas relacionados con la implementación del lenguaje estandarizado en el ejercicio profesional y la formación de las competencias del ser. Conclusiones: se requiere incorporar prácticas pedagógicas en la formación de enfermería que potencien tanto el fortalecimiento disciplinar como las competencias del ser.

Palabras clave: Enfermería; Estudiantes; Educación Basada en Competencias; Anciano; Aprendizaje.

\begin{abstract}
Introduction: Undergraduate nursing training requires the development of pedagogical practices in which aspects are incorporated to promote its characteristics as a profession, discipline, and art. Objective: To analyze the educational experiences in a group of nursing students on the subject of elderly care at a public university in the Los Lagos Region in Chile. Methodology: A clinical assessment guideline was implemented with the use of standardized language and the development of an artistic assignment; at the end of the course, the quantitative-qualitative evaluation of the experience was developed, through a questionnaire and the carrying out of focus groups and individual interviews. Of the 22 students in the course, 18 answered the questionnaire

1. Universidad De Los Lagos. Puerto Montt, Chile.

2. Universidad Austral de Chile. Puerto Montt, Chile.

Correspondencia: Carlos Alberto Fernández Silva. Dirección: Universidad De Los Lagos, Campus Puerto Montt - Chile. Chinquihue km 6. Teléfono: +56966556619. Correo electrónico: carlosalberto.fernandez@ulagos.cl
\end{abstract}


and 8 participated in focus groups and individual interviews. Results: The overall experience was positively valued by the students; it was evidenced that $80 \%$ reported that the assessment guideline used facilitated the application of the nursing process. Regarding the artistic work, the methodological strategies that allowed its development were appreciated, plus the contribution of the work represented for the management of care, the subject and personal areas. Discussion: The contributions that the methodology used could benefit by: solving problems related to the implementation of standardized language in professional practice and the formation of the competencies of being, are evident. Conclusions: It is necessary to incorporate pedagogical practices in nursing training that enhance both disciplinary strengthening and the competencies of being.

Keywords: Nursing; Students; Competency-Based Education; Aged; Learning.

\section{Introducción}

Las metodologías educativas basadas en el paradigma constructivista conllevan al reconocimiento de sus actores, esto es, tanto del docente como facilitador de un proceso activo y de los estudiantes quienes son el eje central del mismo. En este sentido, el proceso de enseñanza-aprendizaje se desarrolla al tomar en consideración el entorno común de sus participantes, además de los conocimientos previos y de otros aspectos como sus intereses y motivaciones, con ello se espera facilitar la adquisición o fortalecer el conocimiento, su deconstrucción, reconstrucción y/o resignificación; de esta manera se aleja de la esterilidad que conlleva el desarrollo de actividades académicas centradas en forma exclusiva en paradigmas conductistas ${ }^{1,2}$.

Los postulados del constructivismo han sido acogidos en la formación universitaria, siendo incorporados no sólo en el ámbito de la pedagogía, sino también en las carreras de salud como la enfermería. La incorporación de metodologías activas como el aprendizaje basado en problemas y casos o la simulación es evidente. Esto es coherente con la actualización de diseños curriculares que responden a las exigencias de calidad en la formación a nivel país ${ }^{3-6}$.

Desde este escenario, las personas dedicadas a la labor docente y académica se enfrentan a la necesidad de evaluar constantemente sus metodologías e incorporar las adecuaciones necesarias para favorecer el aprendizaje, guardando relación con los lineamientos para la formación basada en competencias, las cuales, pese a ser diferentes según los sellos diferenciadores de las instituciones formadoras, para el caso de aquellas que imparten la carrera de enfermería, mantienen un vínculo común relacionado con el deber de resguardar la incorporación de las características epistemológicas que la posicionan como disciplina, profesión y arte ${ }^{7,8}$.

En relación con lo anteriormente descrito, es pertinente mencionar brevemente cada una de dichas características: como disciplina, la enfermería ha logrado consolidarse gracias a sus paradigmas de cuidado y al desarrollo de un lenguaje estandarizado o taxonomías, que permiten establecer el carácter sistemático del ejercicio del cuidar, sea este dirigido a individuos, familias y/o comunidades ${ }^{9,10}$.

Como profesión, la enfermería desarrolla su ejercicio cotidiano de acuerdo con estructuras normativas y legales que conllevan responsabilidades éticojurídicas ${ }^{11,12}$, lo cual motiva el fortalecimiento disciplinar en sus acciones rutinarias de cuidado, y la necesaria evidencia estos a través de parámetros como los registros clínicos.

Respecto al último componente, y no por ello el menos importante, se da el carácter de la enfermería como arte, entendiendo este como un concepto complejo en el que están involucradas las experiencias del profesional de enfermería en su interacción material e inmaterial con el sujeto del cuidado, lo que le permite aproximarse a su compresión, de esta forma mejora tanto su práctica de cuidado como el ejercicio crítico de su profesión ${ }^{13,14}$.

Si bien estas tres características son abordadas durante la formación de los profesionales de enfermería, es común apreciar que el foco de dicho proceso usualmente está centrado en los componentes disciplinar-profesional y con menos fuerza en el estético que conlleva a la incorporación del arte; pese a ello existen experiencias académicas que dan cuenta del interés e importancia de incluirlas en la formación ${ }^{15,16}$.

Desde este contexto, se consideró pertinente incorporar los fundamentos pedagógicos que otorga el constructivismo y en particular, el aprendizaje significativo ${ }^{17}$ en una asignatura en la cual se enseñan los cuidados de enfermería en personas mayores. Es por ello que su aplicación se planeó a través de dos estrategias: la primera, una pauta de valoración clínica basada en los dominios NANDA (North American Nursing Diagnosis Association) $)^{10}$, idea que había sido sugerida por estudiantes en años anteriores para facilitar 
el desarrollo del proceso de enfermería; la segunda estrategia consistió en solicitar a los estudiantes el desarrollo de un ejercicio artístico de libre elección en el que pudiesen vincular elementos del cuidado de enfermería en este grupo etario.

Con estos dos aspectos se espera contribuir a la formación del pensamiento crítico en los estudiantes y por ende, facilitar sus habilidades en la implementación de cuidados. Esto resulta fundamental para que el ejercicio profesional sea activo, se convierta en una práctica que trascienda la formación universitaria y acompañe al futuro profesional de forma cotidiana ${ }^{18-19}$.

Los resultados obtenidos en el transcurso de la asignatura generaron la necesidad de difundirlos con el fin de contribuir a visibilizar las prácticas académicas de enfermería en la formación de pregrado, motivo por el cual se trazó como objetivo general del presente trabajo analizar los resultados de la evaluación realizada a un grupo de estudiantes de enfermería respecto de experiencias formativas en el contexto de una asignatura desde la que se aborda el cuidado de personas mayores en una universidad pública en la Región de Los Lagos en Chile.

\section{Materiales y métodos}

La metodología educativa se aplicó a un curso de IV año de enfermería en la asignatura "Proceso de Enfermería en el Adulto Mayor" en la cual se abordan cuidados geronto-geriátricos de enfermería. Para ello se desarrolló un contenido teórico condensado antes del ingreso a las prácticas institucionales. El curso estuvo compuesto por 22 estudiantes, quienes en su totalidad aplicaron en forma individual la pauta de valoración con base en el NANDA, que fue entregada en forma previa a las prácticas institucionales para ser incorporada en la valoración de personas mayores de un Establecimiento de Larga Estadía para el Adulto Mayor (ELEAM), cuya población se caracteriza por presentar síndromes geriátricos.

Respecto del trabajo artístico, éste fue solicitado como parte del contenido teórico en forma voluntaria al grupo de estudiantes, de los cuales 18 lo llevaron a cabo durante el desarrollo del componente teórico de la asignatura. El trabajo consistió en realizar una producción artística en modalidad libre, por ejemplo: pintura, fotografía, relatos, cuentos, entre otros, según el interés, afinidad y/o habilidades de los estudiantes, a partir de los cuales se pudiera evidenciar aspectos relacionados con el cuidado de enfermería y las personas mayores. La calificación obtenida tributó a quienes lo realizaron en un porcentaje de una de las evaluaciones teóricas principales.

Al finalizar el curso se realizó la evaluación de la asignatura para conocer la opinión y percepciones de los estudiantes. Para darle mayor estructura a este proceso se tomó como base aspectos relacionados con diseños de investigación cuantitativo de tipo descriptivo y cualitativo, por este motivo se invitó a participar a los estudiantes con carácter voluntario, obteniéndose respuesta de 15 de ellos para el componente cuantitativo y 8 para el desarrollo de grupos focales y entrevistas individuales.

Se trazó como objetivos específicos de la evaluación: identificar la opinión de los estudiantes respecto de la pauta de valoración de práctica clínica con base en el NANDA e indagar respecto de su percepción del trabajo artístico desarrollado.

Para cumplir el primer objetivo específico se elaboró un cuestionario de carácter anónimo que contenía preguntas estructuradas y semiestructuradas con opción de respuesta breve, y luego se envió a través de correo electrónico, mediante el uso de la aplicación de "formularios" de Google ${ }^{\circledR}$; esta información posteriormente se analizó empleando frecuencias y porcentajes.

El cuestionario contenía las siguientes preguntas: Respecto de la pauta de valoración de enfermería basada en el NANDA, ¿considera que su aplicación fue más comprensible en comparación con otras pautas de valoración empleadas durante la carrera?; ¿la pauta le facilitó realizar otros componentes del proceso enfermero en general, tales como los diagnósticos y la planificación? Se planteó como opción de respuesta para cada una de ellas: "sí", "no", "fue igual que otras pautas de valoración empleadas anteriormente en otras asignaturas"; para finalizar se les solicitó mencionar brevemente los aspectos positivos y por mejorar.

Para lograr el segundo objetivo se llevó a cabo un grupo focal y tres entrevistas individuales, se tomó como base un cuestionario semiestructurado. La información se recolectó en grabaciones de audio, la cuales fueron transcritas manteniendo el anonimato de los participantes y posteriormente fueron analizadas empleando el software AtlasTi $8^{\circledR}$.

Cabe mencionar que los instrumentos de evaluación de la experiencia fueron de elaboración propia, y que se realizó un proceso de consentimiento informado a 
todos los estudiantes. Se destaca la buena voluntad en la participación, el anonimato y la destrucción del material de audio una vez transcrito, además de la ausencia de represalias o incentivos por parte de los académicos ante su participación, teniendo en cuenta que este ejercicio correspondía a la evaluación de la asignatura como tal. Se trató de evidenciar la experiencia educativa a través de un proceso de evaluación que tomó como referencia aspectos relacionados con diseños de investigación cuantitativos y cualitativos; sin embargo, el propósito del presente texto dista de procesos de investigación.

\section{Resultados}

El $80 \%$ de los estudiantes manifestó que la pauta de valoración fue comprensible y facilitó las etapas de diagnóstico y planificación de cuidados, en comparación con otras pautas empleadas para tal fin (Gráfico 1).

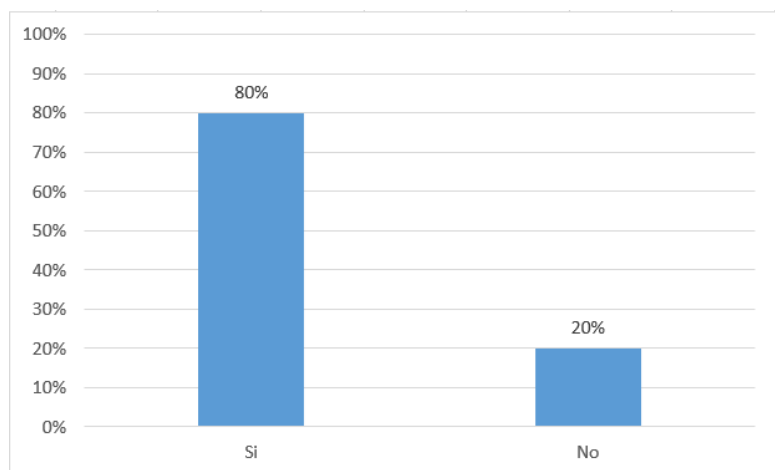

Gráfico 1. ¿La pauta de valoración facilitó las etapas de diagnóstico y planificación?

Fuente: Elaboración propia de los autores.

Entre los aspectos catalogados como positivos de dicha pauta, se destacó el favorecer el orden y continuidad del proceso de enfermería en relación con las etapas de diagnóstico y planificación, lo que posibilita el uso de lenguaje estandarizado y a su vez aporta a la integralidad en la valoración.

El análisis cualitativo resultante de las entrevistas individuales y grupos focales permitió obtener diferentes códigos que fueron organizados en un mapa constituido por dos grandes componentes: las estrategias metodológicas empleadas para la construcción del trabajo artístico y el metaanálisis respecto de la experiencia, término que se empleó para enmarcar los aportes referidos por los participantes en aspectos que trascendieron a la metodología como tal (Ilustración 1).

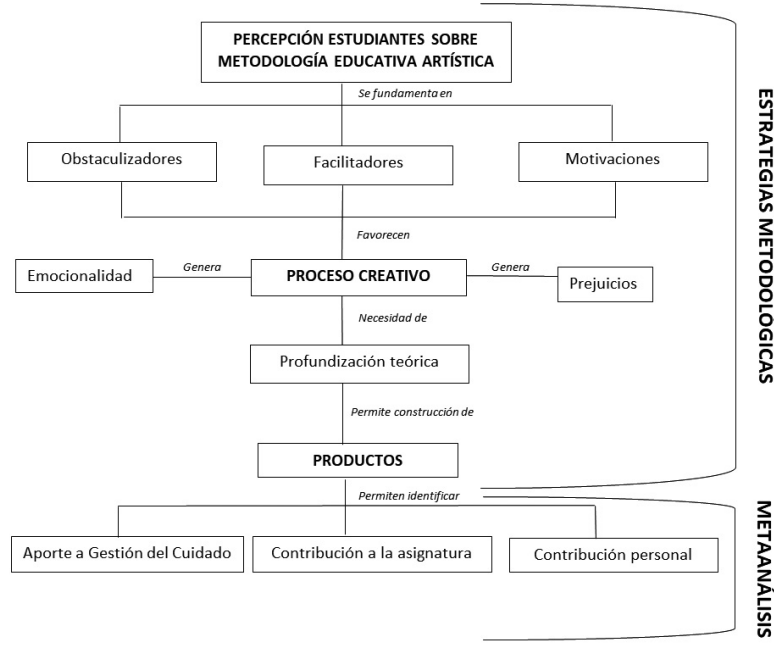

Ilustración 1. Mapa de códigos - análisis cualitativo. Fuente: Elaboración propia de los autores.

En relación con la percepción de los estudiantes sobre la metodología educativa artística, se pudo establecer que ésta se fundamentó en tres ámbitos principales, codificados como "facilitadores", "obstaculizadores" y "motivaciones", siendo las citas más representativas las mencionadas en la Tabla 1.

Tabla 1. Códigos - percepciones.

\begin{tabular}{l|l}
\hline Facilitadores & $\begin{array}{l}\text { Grupo focal: "como me encanta dibujar, el conectar eso con la asignatura, fue como voy a poder dibujar y } \\
\text { sin el sentimiento de que estoy perdiendo el tiempo". }\end{array}$ \\
Obstaculizadores & $\begin{array}{l}\text { Grupo focal: "el tiempo". } \\
\text { "yo no tengo mucha creatividad". } \\
\text { "conectarme con mi yo artístico". } \\
\text { Grupo focal: "sabia que el trabajo iba a llevar una nota, pero ya no era por la nota, era por la gratificación } \\
\text { que me estaba dando realizar el trabajo". }\end{array}$ \\
\hline
\end{tabular}

Fuente: Elaboración propia de los autores.

Cabe mencionar que las motivaciones fueron consideradas como el motor para la realización del trabajo artístico, debido a que favorecieron el proceso creativo en el que se involucraron aspectos como la emocionalidad y la presencia de prejuicios existentes hacia las personas mayores, sumado a la necesidad de profundización teórica respecto de algunos elementos que facilitarían dicho proceso (Tabla 2). 
Tabla 2. Códigos - proceso creativo.

\begin{tabular}{|c|c|}
\hline roceso creativo & $\begin{array}{l}\text { Entrevista: "creo que buscando entre fotografias de mi abuelo como que me surgió la idea de hacer } \\
\text { el dibujo". } \\
\text { Grupo focal: "me motivé mucho y a la semana fui a comprar block, lápices, como no tenía acá, y } \\
\text { buscando un papel especial, porque yo ya habia dibujado antes". }\end{array}$ \\
\hline Emocionalidad & $\begin{array}{l}\text { Grupo focal: "yo creo a todos nos pasó, que mientras haciamos lo que elegimos hacer, se nos venian } \\
\text { recuerdos familiares". } \\
\text { Grupo focal: "el ejercicio que hice fue tratar de conectarme con una persona que para mi fue } \\
\text { bastante especial, que falleció hace un buen tiempo, creo que fue como un trabajo, reitero, bastante } \\
\text { importante y sensible". }\end{array}$ \\
\hline Prejuicios & $\begin{array}{l}\text { Grupo focal: "prejuicios que vimos en la primera clase, que fue ¿cuántas personas están postradas?", } \\
\text { y nosotros dimos cifras muy altas, y en realidad no eran tantas las personas mayores que estaban } \\
\text { postradas, entonces fuimos cambiando muchas realidades que nosotros creíamos". } \\
\text { Grupo focal: "nosotros fuimos derribando, con el paso de la asignatura, como el tema de la } \\
\text { incontinencia, que no tiene por qué ser una incontinencia por la edad, y como que uno siempre } \\
\text { escuchaba a personas mayores que decian que tenían incontinencia y la respuesta era "ya está } \\
\text { viejito", eso ya se fue como derribando, entonces uno puede atender a las personas con otra mirada". } \\
\text { Entrevista: "es como quitarle el estigma a las personas mayores que son como muy dependientes o } \\
\text { aisladas, me favoreció quitarle ese estigma". }\end{array}$ \\
\hline Profundización & $\begin{array}{l}\text { Entrevista: "sí, investigué más sobre el golpe militar, igual lo que uno siempre ve de la televisión y } \\
\text { eso nada más, pero no habia investigado qué pasó". } \\
\text { "estuve leyendo los documentos relacionados con el autocuidado y como se adaptaba la ciudad a } \\
\text { las personas". }\end{array}$ \\
\hline
\end{tabular}

Fuente: Elaboración propia de los autores.

Los trabajos artísticos elaborados por los estudiantes consistieron en construcciones literarias, fotografías, pinturas y dibujos; para estos tres últimos se adjuntó un texto explicativo a fin de comprender el contexto en el cual fueron elaborados (Ilustración 2).

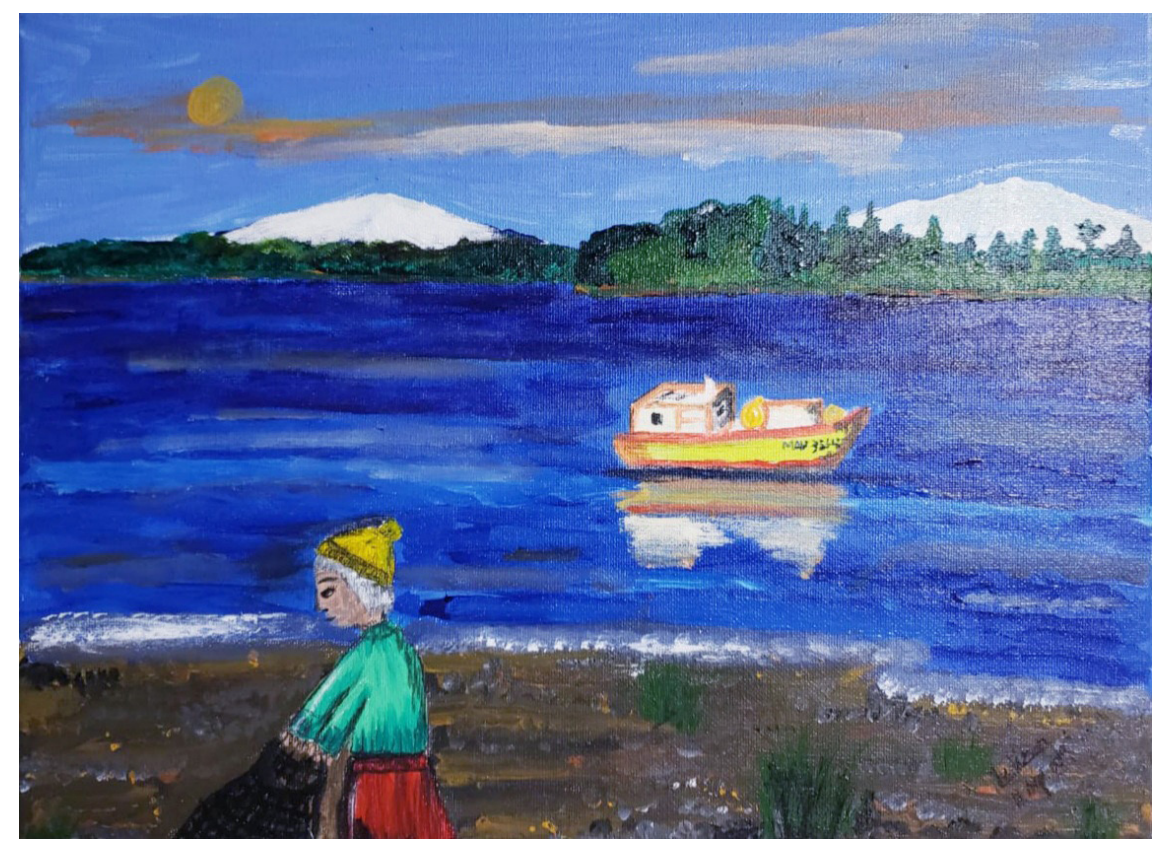

Ilustración 2. Ejemplo de trabajo artístico.

Fuente: Fotografía de uno de los trabajos artísticos realizados por las estudiantes*.

\footnotetext{
*Acrílico sobre tela realizado por la estudiante Ivanna Francesca Vera Soto. Nombre de la obra: El Pasar de tiempo en el sur. Descripción: Pasan los años, la mujer de mar va cambiando, su cuerpo no es el de antes: su marcha se deteriora, pero sus ganas de entregar el sustento a su familia mariscando a orillas del río Maullín no pasan, sus pies se enraízan con la playa, devolviéndose al lugar donde pertenece: el mar.
} 
Con base en estos trabajos se pudo percibir el aporte que representaba para ellos esta metodología hacia la gestión del cuidado, a la asignatura como tal y a otros aspectos de tipo personal, los que fueron agrupados en la categoría de metaanálisis (Tabla 3 ).

Tabla 3. Códigos - metaanálisis.

\begin{tabular}{l|l}
\hline Contribución a la Gestión del cuidado & $\begin{array}{l}\text { Entrevista: "se podría aplicar en talleres de estimulación cognitiva, y sería un buen } \\
\text { aporte para ayudar a las personas a dejar la rutina y favorecer la concentración en } \\
\text { ello [se refiere al usar el dibujo como parte e las intervenciones de enfermería]. Como } \\
\text { futura profesional el aporte de este ejercicio sería como una terapia". } \\
\text { "en la valoración" [identifica el aporte del trabajo artístico dentro del proceso de } \\
\text { enfermería] } \\
\text { Grupo focal: "yo creo que la lucha de muchos estudiantes es sacarse la mirada } \\
\text { biomédica, para mí fue un punto bastante importante, o sea, aún las personas mayores } \\
\text { como que si bien es cierto, son crónicos, tienen adherencia al tratamiento, pero para } \\
\text { ellos es irrelevante aquello, más bien hay un aspecto de sanidad mental emocional, } \\
\text { que ellos lo ponen frente a todas sus prioridades y creo que desde el punto de vista del } \\
\text { cuidado de enfermería, es como que entrega un buen abordaje y reitero como que uno } \\
\text { se trata de despejar esa mirada biomédica". } \\
\text { "sirvió para ser humanos con el trabajo". } \\
\text { Grupo focal: "finalmente, como el trabajo artístico, yo sé que a muchas personas les } \\
\text { pasó, y también a mí, como que empezó a potenciar, como sensibilidades de la forma } \\
\text { de abordar el ramo, más aun, en la práctica clínica creo que fue un desafio bastante } \\
\text { interesante y reitero que fue motivante" } \\
\text { Entrevista: "a mí me gustó eso de dibujar, porque como que me sacó de todo lo que } \\
\text { tenía que realizar, asi que con eso como que me distraje, como que no sé...tuve un } \\
\text { ambiente bueno y estuve relajada en ese momento realizando ese trabajo artístico y } \\
\text { por eso me gustó... ". } \\
\text { Grupo focal: "fue una buena instancia como para desconectarse de todo lo que era la } \\
\text { materia y poder integrar un poco de conocimiento, sin la presión". }\end{array}$ \\
\hline Fución personal
\end{tabular}

Fuente: Elaboración propia de los autores.

\section{Discusión}

La percepción de los estudiantes respecto de la pauta de valoración clínica y su contribución al proceso de enfermería y en particular, hacia el uso de las taxonomías propias de la disciplina, resulta un hallazgo relevante si se tiene en consideración que en el ejercicio rutinario del cuidado de enfermería se han evidenciado dificultades para la aplicación de éstas. Argumentan falta de conocimientos para realizarlo y otros aspectos tales como la alta demanda de cuidados por parte de los usuarios ${ }^{20,21}$.

Cabe mencionar que la aplicación de cuidados que se fundamentan en la sistematización del lenguaje estandarizado contribuye a mejorar la calidad de estos, siendo éste un parámetro fundamental para la actuación de enfermería en el contexto de la gestión del cuidado, y con el cual se aporta además al fortalecimiento de su carácter disciplinar ${ }^{23,24}$.

En relación con la incorporación del arte en las prácticas pedagógicas durante el período de formación de los estudiantes, se puede afirmar que contribuye con su formación integral al favorecer el desarrollo de procesos de comunicación, expresión y reflexión respecto de situaciones personales que se pueden vincular con los cuidados de enfermería. Lo cual permite su identificación como sujetos y aporta la consolidación de las competencias del ser, a partir de las cuales se espera se forje el autoconocimiento y valores como la empatía y solidaridad, que conllevan a la construcción de ciudadanía, de la identidad profesional, y al fortalecimiento de aspectos filosóficos propios de la enfermería como lo son los patrones del conocimiento ${ }^{25-28}$.

La motivación evidenciada en el grupo de estudiantes para el desarrollo de los trabajos fue un factor que favoreció el aprendizaje y la proactividad en la búsqueda de nuevos conocimientos. Por tal razón, resulta importante explorar los elementos que la potencian al ser un aspecto que se considera fundamental dentro del aprendizaje significativo ${ }^{29}$.

La valoración de las didácticas implementadas en la asignatura permite establecer la necesidad de reflexión por parte de los profesionales de enfermería que se dedican a la docencia, respecto de sus prácticas 
pedagógicas y cómo estas incorporan aspectos epistemológicos de su disciplina en el proceso de enseñanzaaprendizaje $^{30}$. Lo que conlleva a la exploración de paradigmas de formación diferentes a los tradicionales que se vinculan con visiones positivistas de la docencia y del currículum, como se ha evidenciado en otras investigaciones ${ }^{31}$.

A partir de esta evaluación, en los estudiantes se hace necesario explorar aspectos como: la percepción de deshumanización de la salud; la visión biomédica, pese a plantearse curricularmente el abordaje de paradigmas integrales de cuidado, y sobre la gestión del tiempo de estudio, teniendo en consideración lo referido en cuanto a la percepción de sobrecarga académica. Por último, se considera pertinente incorporar este tipo de metodologías en otras asignaturas y niveles de estudio.

\section{Conclusiones}

El profesional de enfermería que se dedica a la docencia debe propender por la incorporación de prácticas pedagógicas que permitan relevar la generación de cuidados fundamentados en aspectos disciplinares y epistemológicos que les son propios, además de fomentar la reflexión respecto del aporte que éstas realizan a la formación de las competencias del ser.

Se puede apreciar la necesidad de contar con asesoría para la implementación curricular en enfermería de tal manera que se garantice los aspectos anteriormente comentados, además de generar las alianzas respectivas con profesiones como la pedagogía en artes, con el fin de elaborar instrumentos que permitan la evaluación del ejercicio artístico.

Se reconoce como limitaciones de este estudio, haber omitido la percepción de los cuatro estudiantes que no desarrollaron el trabajo artístico, además se destaca que esta investigación corresponde una innovación educativa. Por tal razón, los datos presentados no pretender establecer generalizaciones o inferencias ya que su intención es evidenciar prácticas exitosas pedagógicas con enfoque disciplinar.

\section{Agradecimientos}

A los estudiantes de IV año de la Universidad De Los Lagos II semestre de 2019 del campus Puerto Montt, Región De Los Lagos - Chile, por su motivación y por su disposición para incorporar nuevas ideas en el proceso de enseñanza-aprendizaje.

\section{Consideraciones éticas}

Pese a ser una innovación educativa, para el abordaje del presente trabajo se aplicó medidas para el resguardo de la confidencialidad y anonimato de las personas que participaron en la evaluación de la asignatura, motivo por el que se realizó el proceso de consentimiento informado, en el cual se solicitó la autorización para el uso de la información en el presente texto.

\section{Conflicto de intereses}

Los autores declaran no tener ningún conflicto de interés.

\section{Referencias}

1. Agra G, Formiga N, Oliveira P, Costa M, Fernandes M, Nóbrega MM. Análisis del concepto de aprendizaje significativo a la luz de la teoría de Ausubel. Rev Bras Enferm. 2019; 72(1): 248-255. http://dx.doi.org/10.1590/0034-7167-2017-0691

2. Ordoñez Olmedo E, Mohedano Sánchez I. El aprendizaje significativo como base de las metodologías innovadoras. Rev Educ Hekademos. 2019; 26: 18-30

3. Darroz LM. Aprendizagem significativa: a teoria de David Ausubel. Esp Pedagóg. 2018; 25(2): 576-580. doi: https://doi.org/10.5335/rep.v25i2.8180

4. Sousa ATO de, Formiga NS, Oliveira SH dos S, Costa MML, Soares MJGO. El uso de la teoría del aprendizaje significativo en la educación de enfermería. Rev Bras Enferm. 2015; 68(4): 713-22. doi: https://doi.org/10.1590/0034$7167.2015680420 \mathrm{i}$

5. del Carmen Castillo-Arcos L, Maas-Góngora L. Percepción de satisfacción de los estudiantes de enfermería en el uso de la simulación clínica. Ra Ximhai. 2017; 13(2): 63-76.

6. Leal Oyarzún M, Illesca Pretty M, González Osorio L. Estrategias metodológicas de enseñanzaaprendizaje en estudiantes de enfermería: mejorando la calidad de la formación profesional. Dilemas Contemporáneos: Educación, Política y Valores 2018; 5(2): 1-16.

7. Morales Iturio A. El Constructivismo como fundamento de la Práctica Docente de Enfermería en el contexto de la Formación por Competencias. Dilemas Contemporáneos: Educación, Política y Valores 2017; 5(1): 1 .

8. Cancino Cancino V, Iturra Herrera C. Gestión curricular en un enfoque por competencias: aspectos clave y avances en el sistema universitario chileno. 
En: La formación por competencias en la educación superior: alcances y limitaciones desde referentes de México, España y Chile. Ciudad de México: Tirant Humanidades, 2018. p 323-348.

9. Alligood M y Marriner-Tomey A. Modelos y Teorías en Enfermería. $8^{\mathrm{a}}$ ed. Barcelona: Elsevier Mosby, 2014.

10. Hederman H y Kamitsuru S [Eds.]. Diagnósticos enfermeros. Definiciones y clasificación 2015-2017. España: Elsevier.

11. Código Sanitario. Decreto con fuerza de Ley No. 725. Santiago, Chile: s.n., 11 de diciembre de 1967. Última modificación: 23 de septiembre de 2017.

12. Norma técnica general administrativa No. 19. Gestión del Cuidado de Enfermería para la atención Cerrada. Exenta No. 1127. Santiago, Chile: s.n., 14 de diciembre de 2007.

13. Rodríguez S, Cárdenas M, Pacheco AL, Ramírez M, Ferro N, Alvarado E. Reflexión Teórica sobre el arte del cuidado. Enf Univ 2017; 14 (3): 191-198.

14. Rodríguez-Jiménez S, Cárdenas-Jiménez M, Pacheco- Arce AL, Ramírez-Pérez M. Una mirada fenomenológica del cuidado de enfermería. Enf Univ 2014; 11(4): 145-153.

15. Ruiz-Mata F, Ruiz-Bueno C, Aradilla-Herrero A, Edo-Gual M. Otra manera de enseñar enfermería clínica: el arte como vehículo formador. Educ Med. 2018; 19(3): 366-374. https://doi.org/10.1016/j. edumed.2017.07.016

16. Silva O, Alves ED, Rodrigues, MCS. Liricidad y toque de arte para la producción del conocimiento estético de enfermería - una reflexión poética inspirada en la Teoría de la Complejidad. Cult. Cuid. 2014; 18 (39): 14-29. doi: http://dx.doi.org/10.7184/ cuid.2014.39.03

17. Moreira MA. Aprendizaje significativo como un referente para la organización de la enseñanza. Archivos de Ciencias de la Educación 2017; 11(12), e029. doi: https://doi.org/10.24215/23468866e029

18. Alfaro-LeFevre R. Pensamiento crítico, razonamiento clínico, y juicio clínico en enfermería: un enfoque práctico. 6ta ed. España: Elsevier, 2017.

19. Escobar-Castellanos B, Jara Concha P. Filosofía de Patricia Benner, aplicación en la formación de enfermería: propuestas de estrategias de aprendizaje. Educación 2019; (54):182. doi: https:// doi.org/10.18800/educacion.201901.009

20. Ponti LE, Castillo Benites RT, Vignatti RA, Monaco ME, Nuñez JE. Conocimientos y dificultades del proceso de atención de enfermería en la práctica. Educ Med Super. 2017; 31(4): 1-12.
21. Boaventura AP, Dos Santos PA, Marocco Duran EC. Conocimiento teórico-práctico del enfermero del Proceso de Enfermería y Sistematización de Enfermería. Enferm glob 2017; 16 (46): 182-216. doi: http://dx.doi.org/10.6018/eglobal.16.2.247911

22. Campos C, Jaimovich S, Wigodski J, Aedo V. Conocimientos y uso clínico de la metodología enfermera (NANDA, NIC, NOC) en enfermeras/ os que trabajan en Chile. Rev Iberoam Educ Inv Enferm. 2017; 7(1): 33-42.

23. Siles González J. La utilidad práctica de la Epistemología en la clarificación de la pertinencia teórica y metodológica en la disciplina enfermera. Index Enferm 2016; 25(1-2): 86-92.

24. Pérez-Fuillerat N, Solano-Ruiz MC, Amezcua M. Conocimiento tácito: características en la práctica enfermera. Gac Sanit 2019; 33 (2): 191-196. doi: https://doi.org/10.1016/j.gaceta.2017.11.002

25. Lagoueyte Gómez MI. El cuidado de enfermería a los grupos humanos. Rev Univ Ind Santander Salud. 2015; 47(2): 209-213.

26. Bernal Martínez EM, Martín Estévez L, Acosta Gaibor MP, Mazacón Roca BN. Florence Nightingale y la formación de valores en la Enfermería. Dilemas Contemporáneos: Educación, Política y Valores 2019; 6: 1-18.

27. González-Aguilar A, Vázquez-Cataño F, AlmazánTlalpan B, Morales-Nieto A, García-Solano B. Proceso de aprehensión de identidad profesional en enfermería. Rev Cuidarte 2018; 9(3): 2297-2308. doi: http://dx.doi.org/10.15649/cuidarte.v9i3.519

28. Escobar Castellanos B, Sanhueza Alvarado O. Patrones de conocimiento de Carper y expresión en el cuidado de enfermería. Enfermería: cuidados humanizados 2018; 7(1): 27-42. http://dx.doi. org/10.22235/ech.v7i1.1540

29. Agama-Sarabia A, Crespo-Knopfler S. Modelo Constructivista y tradicional: influencia sobre el aprendizaje, estructuración del conocimiento y motivación en alumnos de enfermería. Index Enferm 2016; 25 (1-2): 109-113.

30. Moreno Mojica CM, Barragán Becerra JA. Prácticas pedagógicas y procesos de aprendizaje: configuración e institucionalización en la disciplina de enfermería. Ánfora 2019; 26(46):163-85. doi: https://doi.org/10.30854/anf.v26.n46.2019.559

31. Martínez JC. Practicas evaluativas del proceso de atención de enfermería: una visión de docentes y estudiantes. Rev Cuid. 2017; 8(1): 1459-1475. doi: http://dx.doi.org/10.15649/cuidarte.v8i1.351 